\title{
Effects of prostatic inflammation on LUTS and alpha blocker treatment outcomes
}

\author{
Ha Na Lee ${ }^{1}$, Tae-Hyoung Kim², Sun-Ju Lee ${ }^{3}$, Won Yeol Cho ${ }^{4}$, Bong Suk Shim ${ }^{5}$ \\ ${ }^{1}$ Department of Urology, Ewha Womans University Medical Center Seoul Seonam Hospital; ${ }^{2}$ Chung-Ang \\ University Hospital, Chung-Ang University College of Medicine; ${ }^{3}$ Kyung Hee University Hospital, Kyung \\ Hee University School of Medicine; ${ }^{4}$ Dong-A University Hospital, Dong-A University School of Medicine, \\ Busan and ${ }^{5}$ Ewha Womans University Mok-dong Hospital, Ewha Womans University School of Medicine, \\ Seoul, Korea
}

\section{ABSTRACT}

Purpose: To evaluate the association between prostatic inflammation and lower urinary tract symptoms (LUTS), and to identify the effects of prostatic inflammation on the treatment with an alpha blocker.

Materials and Methods: 111 Participants who were aged $\geq 50$ years, the presence of LUTS (maximal flow rate $<20 \mathrm{~m} / \mathrm{s}$, IPSS $\geq 11$ ), and an elevated PSA level (3-20ng/mL) were treated with tamsulosin $0.2 \mathrm{mg}$ once daily for 3 months after prostate biopsies. Prostatic inflammation was scored as none (0), mild (I), moderate (II), or marked (III). LUTS parameters including urine flow rates, IPSS, PSA, and prostate volume were evaluated. Results: Inflammation grading resulted in 25, 60, and 26 patients that were grade $0, \mathrm{I}$, and II, respectively. Lower grade inflammation was related to higher urine flow rate at baseline. Patients with higher inflammation grades had larger prostate volumes, larger total and transitional zone volumes, and higher PSA levels. Overall, urine flow rates and residual urine volume were improved after 3 months of alpha blocker therapy. Eighty percent of patients with grade 0 inflammation, 73\% of patients with grade I inflammation, and 92.3\% of patients with grade II inflammation showed improvement of LUTS after treatment. Longer duration of treatment was related to a decreased chance of improvement of LUTS. Patients with increased IPSS voiding subscales could be predictive of improvement of LUTS.

Conclusions: Patients with high grade inflammation had lower flow rates and higher prostatic volumes than patients with low grade inflammation. Inflammation grade did not affect the outcomes of alpha blocker treatment.

\section{ARTICLE INFO}

\section{Key words:}

Prostatitis; Prostatic Hyperplasia; Therapeutics; Adrenergic alphaAntagonists

Int Braz J Urol. 2014; 40: 356-66

Submitted for publication:

August 13, 2013

Accepted after revision: January 15, 2014

\section{INTRODUCTION}

Although the pathogenesis of benign prostatic hyperplasia (BPH) is not yet completely understood, there is some evidence that prostatic inflammation could be a key contributor to prostate enlargement and progression of $\mathrm{BPH}$. The presence of chronic histologic inflammation is a common finding in prostatic tissue on biopsies of surgical specimens from patients with and without lower urinary tract symptoms (LUTS) or prostatitis (1-3). Although it is not yet defined when and why chronic inflammation occurs, it has been hypothesized that $\mathrm{BPH}$ is an immune-mediated inflammatory 
disease $(2,4,5)$. Various growth factors and cytokines including $\mathrm{T}$ and $\mathrm{B}$ lymphoid cells and macrophages have been shown to be involved in both the inflammatory process and in the interactions between epithelial and stromal prostatic cells (2).

Several studies have investigated the relationship between histologic prostatic inflammation and LUTS related BPH (6-8). Chronic prostate inflammatory infiltrates were at a higher risk of BPH progression and acute urinary retention when compared with patients without inflammatory infiltrates at baseline. Depending on the relationship between prostatic inflammation and LUTS, medications with anti-inflammatory effects could be a novel treatment option for the management of BPH related LUTS. We evaluated the association between prostatic inflammation and LUTS. We also characterized the effects of prostatic inflammation on the treatment of LUTS with an alpha blocker.

\section{MATERIALS AND METHODS}

\section{Study participants}

The protocol and procedures used in this study were approved by the Institutional Review Board at Ewha Medical Center. Data was retrospectively collected from 10 hospitals in Korea. Patients who met the following criteria were included in the study: (i) men, aged $\geq 50$ years, (ii) total IPSS $\geq 11$, (iii) maximum flow rate (MFR) $<20 \mathrm{~mL} / \mathrm{sec}$. and voided volume $\geq 120 \mathrm{~mL}$, (iv) total prostate volume between 20 and 50g, (v) baseline serum prostate specific antigen (PSA) level between 3 and 20ng/ $\mathrm{mL}$, and (vi) pathologically confirmed $\mathrm{BPH}$ on tissue obtained via transrectal ultrasound-guided biopsy. Exclusion criteria for this study included: (i) history of 5-alpha reductase inhibitor or alpha1-blocker use or a history of surgical therapy for BPH within 6 months of this study, (ii) neurogenic bladder, (iii) known history of an urothelial tumor such as prostate cancer or bladder cancer, and history of a urethral stricture or bladder neck obstruction, (iv) history of orthostatic hypotension with syncope, (v) history of chronic prostatitis/chronic prostatic pain syndrome, (vi) history of acute bacterial prostatitis within 6 months of this study, (vii) history of acute urinary tract infection within 1 month of this study, and (viii) history of unstable angina, myocardial infarction, or cerebro-vascular disease within 6 months of this study.

\section{Evaluations}

All data included in this study were obtained from patients who underwent transrectal ultrasound-guided biopsy of the prostate to rule out prostate cancer. All patients were treated with tamsulosin $2 \mathrm{mg}$ once daily for 3 months post-biopsy. Inflammation grades were reviewed by a single pathologist. The pathologist assessed the prostatic strips throughout whole specimens. Inflammation was assessed across all cores. Scoring of inflammation was based on a histologic grading system as follows: (i) grade 0, no inflammation; (ii) grade I, scattered inflammatory cell infiltrate without nodules; (iii) grade II, no confluent lymphoid nodules; (iv) grade III, large inflammatory areas with confluence (9). We assessed all specimens for degree of inflammation according to the most frequent and most severe scores.

The primary endpoint was the difference in total IPSS according to the prostatic inflammation grade. Secondary endpoints were the differences in IPSS subscales and quality of life scores with respect to prostatic inflammation grade. Baseline LUTS parameters including uroflowmetry data, IPSS, PSA, and prostate volume were evaluated for an association with prostatic inflammation. Uroflowmetry data included voided volume, maximum flow rate, average flow rate, and post-void residual urine (PVR) volume. IPSS was evaluated using a total index score (items 1-7), storage subscale (items 2,4,7), voiding subscale (items 1,3,5,6), and a quality of life item. Total and transitional zone prostate volumes and serum PSA levels were analyzed according to the prostatic inflammation grade. The effect of alpha blocker therapy for LUTS was evaluated according to the inflammation grade. The changes in total IPSS and subscales in the storage and voiding domains, quality of life (QoL) index, MFR, AFR, and post-void residual urine volume were measured at baseline and at 3 months. Improvement was defined as an increase in maximum flow rate $>3 \mathrm{~m} / \mathrm{s}$ or $>25 \%$ improvement in total IPSS. Safety parameters monitored included changes in systolic and diastolic blood pressures, and serious adverse events were recorded. 


\section{Statistical analysis}

We assessed differences in clinical parameters such as MFR, average flow rate (AFR), PVR, IPSS, prostate volume, and PSA level using paired t-tests or Wilcoxon signed-rank tests with Bonferroni correction. We analyzed differences in clinical parameters according to the inflammation grade using Kruskal-Wallis tests and Wilcoxon rank-sum tests with Bonferroni adjustment. All analyses were performed using SAS software version 9.1.3 (SAS Institute Inc., Cary, NC, USA).

\section{RESULTS}

\section{Participants}

A total of 111 patients from 10 hospitals were included in this study. Inflammation grades were assigned based on the most frequent score, with 25 patients at grade 0,60 at grade I, and 26 at grade II. The mean age of each group was 64.3 (grade 0), 65.6 (grade I), and 64.9 years (grade II). The duration of treatment for each group was 6.9, 5.6, and 4.5 months (grades 0, I, and II, respectively). There were no significant differences in demographic data between the groups (Table-1).

Baseline LUTS parameters and prostatic inflammation grades

Differences in baseline LUTS parameters according to inflammation grade were evaluated. The mean MFR of each group was 13.5 (grade 0), 11.9 (grade I), and 10 (grade II). The mean AFR of each group was 8 (grade 0), 6.2 (grade I), and 5.1 (grade II). Lower grade inflammation was associated with higher MFR and AFR ( $<$ 0.05). Mean total prostate volume in each group was 36.3 (grade 0), 38.9

Table 1 - Demographic data of study participants (mean [SD], median [IQR]).

\begin{tabular}{|c|c|c|c|c|}
\hline & $\begin{array}{l}\text { Grade } 0^{*} \\
(\mathrm{~N}=25)\end{array}$ & $\begin{array}{l}\text { Grade I* } \\
(\mathrm{N}=60)\end{array}$ & $\begin{array}{l}\text { Grade } I^{*} \\
(\mathrm{~N}=26)\end{array}$ & $p$-value ${ }^{* *}$ \\
\hline \multicolumn{5}{|l|}{ Age } \\
\hline Mean (SD) & $64.3(7.4)$ & $65.6(7)$ & $64.9(8)$ & \multirow{2}{*}{0.658} \\
\hline Median (IQR) & $64(60,68)$ & $66(61,69.5)$ & $68(58,70)$ & \\
\hline \multicolumn{5}{|l|}{ BMI } \\
\hline Mean (SD) & $23.8(3.5)$ & $23.5(2.8)$ & $24.9(3.3)$ & \multirow{2}{*}{0.265} \\
\hline Median (IQR) & $23.5(22.4,24.8)$ & $23.4(21.4,25.2)$ & $24.4(22.4,27.7)$ & \\
\hline \multicolumn{5}{|l|}{ Systolic BP } \\
\hline Mean (SD) & $123.4(12.5)$ & $123(11.3)$ & $124.6(13.8)$ & \multirow{2}{*}{0.983} \\
\hline Median (IQR) & $125(110,130)$ & $120(120,130)$ & $121(117,130)$ & \\
\hline \multicolumn{5}{|l|}{ Diastolic BP } \\
\hline Mean (SD) & $78.1(9.1)$ & $77.3(8.9)$ & $77.3(12)$ & \multirow{2}{*}{0.832} \\
\hline Median (IQR) & $80(70,85)$ & $78.5(70,82)$ & $80(70,84)$ & \\
\hline \multicolumn{5}{|c|}{ Treatment duration (months) } \\
\hline Mean (SD) & $6.9(7.1)$ & $5.6(10.7)$ & $4.5(5)$ & \multirow[t]{2}{*}{0.092} \\
\hline Median (IQR) & $4(3,7)$ & $3(3,3.8)$ & $3.3(3,4)$ & \\
\hline $\mathrm{DM}(\mathrm{n}[\%])$ & $4(16.0)$ & $2(3.3)$ & $5(19.2)$ & 0.022 \\
\hline Hypertension (n[\%]) & $8(32.0)$ & $11(18.3)$ & $3(11.5)$ & 0.170 \\
\hline CVA $(n,[\%])$ & $3(12.0)$ & $2(3.3)$ & $0(0)$ & 0.121 \\
\hline
\end{tabular}

$\mathbf{S D}=$ standard deviation; $\mathbf{I Q R}=$ interquartile range. * Inflammation grade was based on the most frequent score assigned to prostate specimens. ${ }^{* *}$ Statistical analysis using chi-squared tests. 
(grade I), and 47.9 (grade II). Higher grade inflammation was associated with larger prostate volumes including total and transitional zone volumes $(\mathrm{p}<$ 0.05). Baseline IPSS and QoL scores were unrelated to prostatic inflammation grade. In terms of serum PSA, patients with higher inflammation grades had significantly higher PSA levels $(\mathrm{p}<0.05)$ (Table-2).

Alpha blocker treatment effects according to inflammation grade

We evaluated changes in clinical parameters and symptom scores according to inflammation grade after 3 months of treatment with an alpha blocker (Table-3). Voided volume, MFR, AFR, and PVR improved after 3 months of alpha blocker therapy, although these changes were unrelated to inflammation grade ( $p>0.05)$. Similarly, IPSS improved after alpha blocker treatment, independent of inflammation grade.

The association between inflammation grade and symptomatic improvement

We evaluated whether or not improvement of LUTS was related to inflammation grade. According to the most popular inflammation scoring system, $80 \%$ of patients with grade 0 prostatic inflammation experienced improvement in their LUTS. Seventy-three percent and 92.3\% of patients with grade I and grade II inflammation, respectively, had improvement of LUTS. There was no significant difference in improvement rates among the three groups $(\mathrm{p}=0.1363)$. According to the most stringent inflammation scoring system, $82 \%$ of patients who had grade 0 inflammation experienced improvement. Additionally, 72\%, $83.7 \%$, and $100 \%$ of patients with grade I, II, and III inflammation, respectively, had improvement of LUTS ( $p=0.3185$ ) (Table-4).

Predictive factors for treatment responses to alpha blockers

Factors that predicted symptomatic improvement as a result of alpha blocker therapy were evaluated using univariate and multivariate analysis. Longer duration of medication use was related to decreased symptomatic improvement (OR $=0.92$, $95 \%$ CI 0.85 - 0.99). Increased IPSS voiding subscales were associated with an increase in symptoma- tic improvement $(\mathrm{OR}=1.17,95 \%$ CI $1.02-1.34)$. Thus, more severe symptoms, which were represented by high IPSS voiding scores, could be predictive of improvement of LUTS with alpha blocker therapy (Tables 5 and 6).

\section{Safety}

Systolic blood pressures were decreased by a mean of $4.3 \mathrm{mmHg}$ after 3 months of treatment with an alpha blocker compared to baseline, while diastolic blood pressures were decreased by a mean of $4.7 \mathrm{mmHg}$. There were no serious adverse events related to treatment with tamsulosin.

\section{DISCUSSION}

The present study sought to evaluate whether there is a correlation between histologically graded prostatic inflammation and prostate-related lower urinary tract symptoms. Our data suggest that prostatic inflammation grades are associated with LUTS, and that high grade inflammation was associated with lower urine flow rates and higher prostate volumes than low grade inflammation.

Prostatic inflammation is gaining increasing attention as a potential etiologic factor in prostate cancer, benign prostatic hyperplasia, lower urinary tract symptoms, and chronic pelvic pain syndrome (CPPS). In a mouse model, acute bacterial inflammation of the prostate was associated with epithelial proliferation and reactive hyperplasia (10). This study concluded that transurethral inoculation of uropathogenic E.coli 1677 reliably infected the mouse prostate, produced a significant inflammatory response, and induced quantifiable epithelial proliferation and reactive hyperplasia.

Similar to the intestine and the lung, the prostate is considered to be an immune-competent organ. It is populated by a small number of inflammatory cells (leukocytes) that increase with age and consist of scattered stromal and intraepithelial $\mathrm{T}$ and $\mathrm{B}$ lymphocytes, macrophages, and mast cells. Several reports have evaluated the constituents of inflammatory infiltrates in patients with BPH. The REDUCE study reported that chronic inflammation is observed in $77.6 \%$ of patients with LUTS, and that the higher the average chronic inflammation score, the higher the IPSS. In the 
Table 2 - Differences in baseline LUTS parameters according to inflammation grade.

\begin{tabular}{|c|c|c|c|c|}
\hline & $\begin{array}{l}\text { Grade } 0 \\
(\mathrm{~N}=25)\end{array}$ & $\begin{array}{l}\text { Grade I } \\
(\mathrm{N}=60)\end{array}$ & $\begin{array}{l}\text { Grade II } \\
(\mathrm{N}=26)\end{array}$ & $p$-value \\
\hline \multicolumn{5}{|l|}{ Uroflowmetry } \\
\hline \multicolumn{5}{|c|}{ Voided volume (mL) } \\
\hline Mean (SD) & $236.9(108.3)$ & $223(85.4)$ & $194.1(106.3)$ & \multirow{2}{*}{0.148} \\
\hline Median (IQR) & $184.1(160,294)$ & $196.5(161,269.7)$ & $170.4(129,217)$ & \\
\hline \multicolumn{5}{|c|}{ Maximum flow rate (mL/s) } \\
\hline Mean (SD) & $13.5(3.5)$ & $11.9(3.6)$ & $10(3.3)$ & \multirow{2}{*}{$0.005^{\star}$} \\
\hline Median (IQR) & $15(10,16.3)$ & $11.9(9.2,14.9)$ & $10(7,12.3)$ & \\
\hline \multicolumn{5}{|c|}{ Average flow rate (mL/s) } \\
\hline Mean (SD) & $8(2.9)$ & $6.2(2.8)$ & $5.1(2.2)$ & \multirow{2}{*}{$0.002^{*}, \dagger$} \\
\hline Median (IQR) & $8(5.9,10.3)$ & $5.5(4.2,8)$ & $4.8(3.7,6.7)$ & \\
\hline \multicolumn{5}{|c|}{ Post-void residual urine volume (mL) } \\
\hline Mean (SD) & $59.6(55.2)$ & $57.3(56.5)$ & $52.8(50.3)$ & \multirow{2}{*}{0.615} \\
\hline Median (IQR) & $47(25,80)$ & $43(18.5,79)$ & $(31,20,87)$ & \\
\hline \multicolumn{5}{|l|}{ IPSS } \\
\hline \multicolumn{5}{|l|}{ Total index score } \\
\hline Mean (SD) & $14.8(5.3)$ & $17.2(7)$ & $17.4(4.4)$ & \multirow{2}{*}{0.088} \\
\hline Median (IQR) & $14(12,17)$ & $15.5(12,21)$ & $16.5(14,21)$ & \\
\hline \multicolumn{5}{|l|}{ Storage subscale } \\
\hline Mean (SD) & $5.9(2.6)$ & $6.8(3.1)$ & $7.2(2.3)$ & \multirow{2}{*}{0.160} \\
\hline Median (IQR) & $6(4,7)$ & $6(5,8.5)$ & $7(6,8)$ & \\
\hline \multicolumn{5}{|l|}{ Voiding subscale } \\
\hline Mean (SD) & $8.8(3.9)$ & $10.4(4.8)$ & $10.2(4.4)$ & \multirow{2}{*}{0.174} \\
\hline Median (IQR) & $8(7,9)$ & $10(7,14)$ & $9.5(7,14)$ & \\
\hline \multicolumn{5}{|l|}{ Quality of life item } \\
\hline Mean (SD) & $3.2(1.2)$ & $3.5(1.1)$ & $3.6(1.1)$ & \multirow{2}{*}{0.391} \\
\hline Median (IQR) & $3(3,4)$ & $4(3,4)$ & $4(3,4)$ & \\
\hline \multicolumn{5}{|l|}{ Prostate volume } \\
\hline \multicolumn{5}{|l|}{ Total volume (cc) } \\
\hline Mean (SD) & $36.3(8.2)$ & $38.9(10.7)$ & $47.9(26.5)$ & \multirow{2}{*}{$0.018^{\star}, \ddagger$} \\
\hline Median (IQR) & $37(32,43)$ & $38(30.2,46)$ & $41.7(31.3,57)$ & \\
\hline \multicolumn{5}{|c|}{ Transitional zone volume (cc) } \\
\hline Mean (SD) & $15(7.8)$ & $19(8.5)$ & $27.3(19.6)$ & \multirow{2}{*}{$0.001^{*}, \ddagger$} \\
\hline Median (IQR) & $11.1(9.8,19)$ & $17.3(12.6,25.7)$ & $23(13.9,37)$ & \\
\hline \multicolumn{5}{|l|}{ PSA (ng/mL) } \\
\hline Mean (SD) & $6.3(4.6)$ & $7.6(11.6)$ & $8.4(4.7)$ & \multirow{2}{*}{0.027} \\
\hline Median (IQR) & $4.4(3.7,6)$ & $5.3(4.6,6.8)$ & $8(4.6,10.9)$ & \\
\hline
\end{tabular}

SD = standard deviation; IQR = interquartile range. *Significant difference between grades 0 and II, $†$ significant difference between grades 0 and I, $\ddagger$ significant difference between grades I and II. Kruskal-Wallis tests using Wilcoxon rank-sum tests with Bonferroni adjustment. 


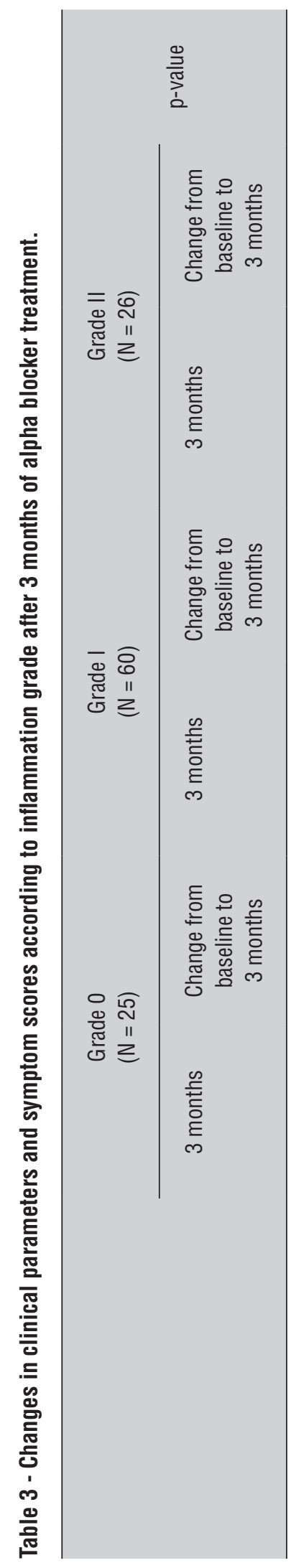

స్

్ֶ?

$\underset{\substack{\mathrm{o} \\ 0}}{\circ}$

مิ

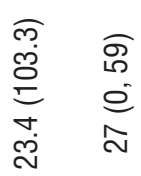

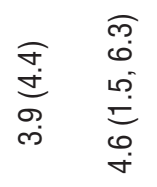

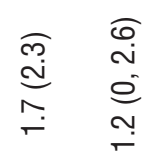

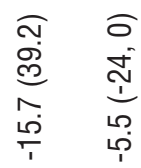

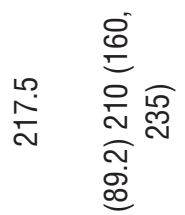

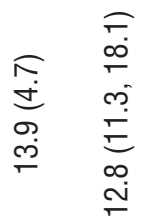

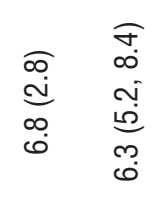

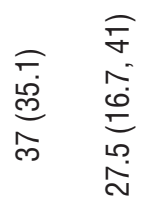

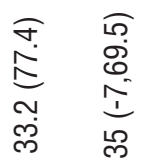

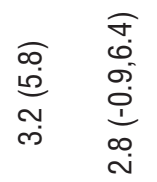

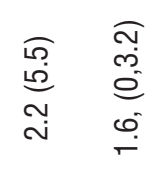

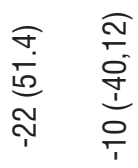

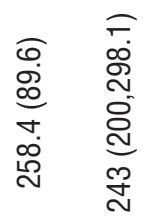

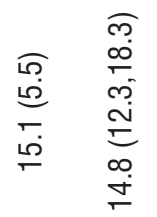

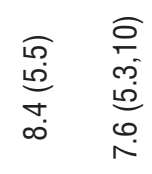

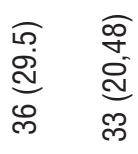

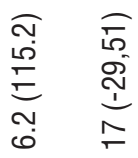

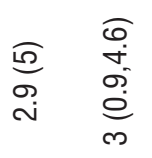

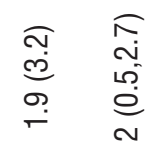

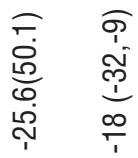

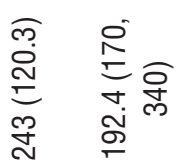

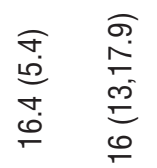

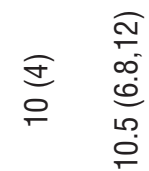

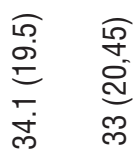

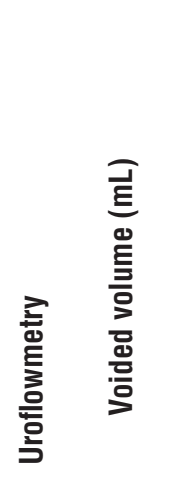

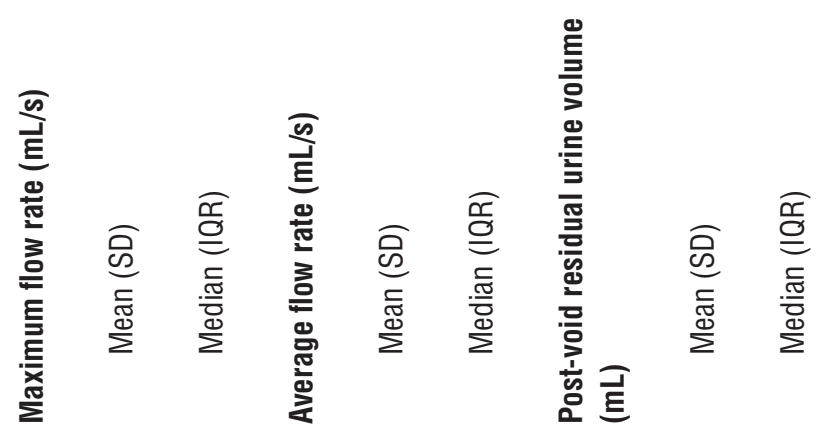


ָัญ

$\stackrel{\infty}{\infty}$

$\underset{\substack{\Re \\ 0}}{\stackrel{m}{0}}$

惢

હ̃

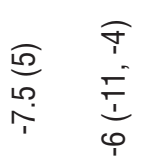

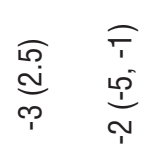

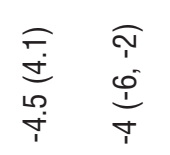

$\begin{array}{ll}\mathscr{1} & 0 \\ \check{T} & \stackrel{1}{T}\end{array}$

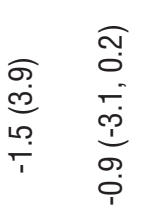

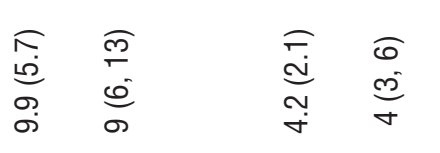

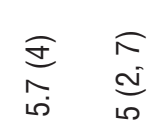

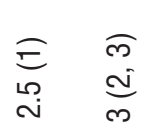

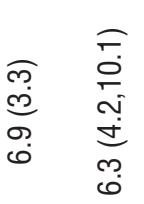

\begin{tabular}{cc} 
& 0 \\
0 & 0 \\
0 & 1 \\
0 & 0 \\
0 & 0 \\
0 & 0 \\
\hline 1 & 0
\end{tabular}

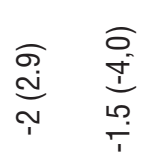

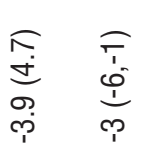

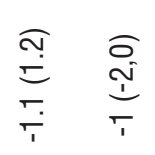

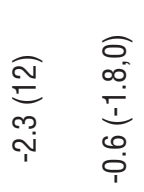

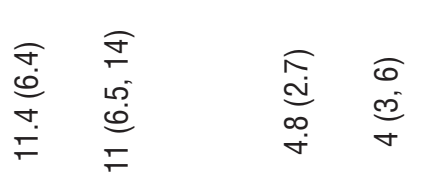

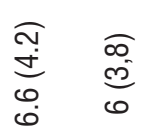

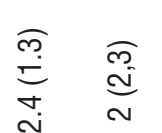

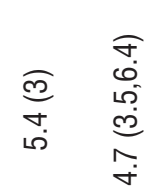

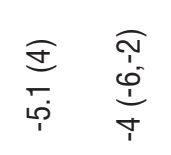

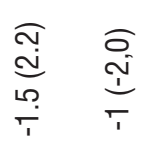

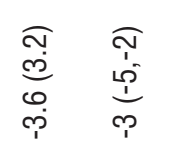

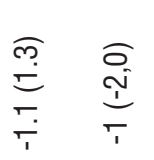

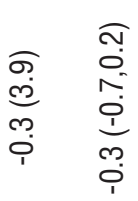

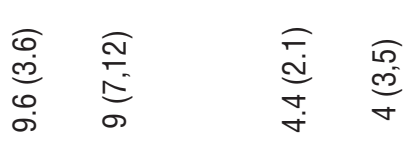

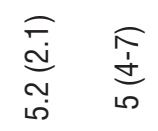

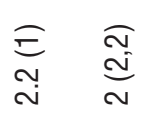

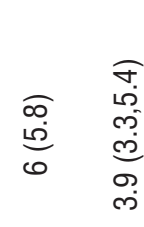

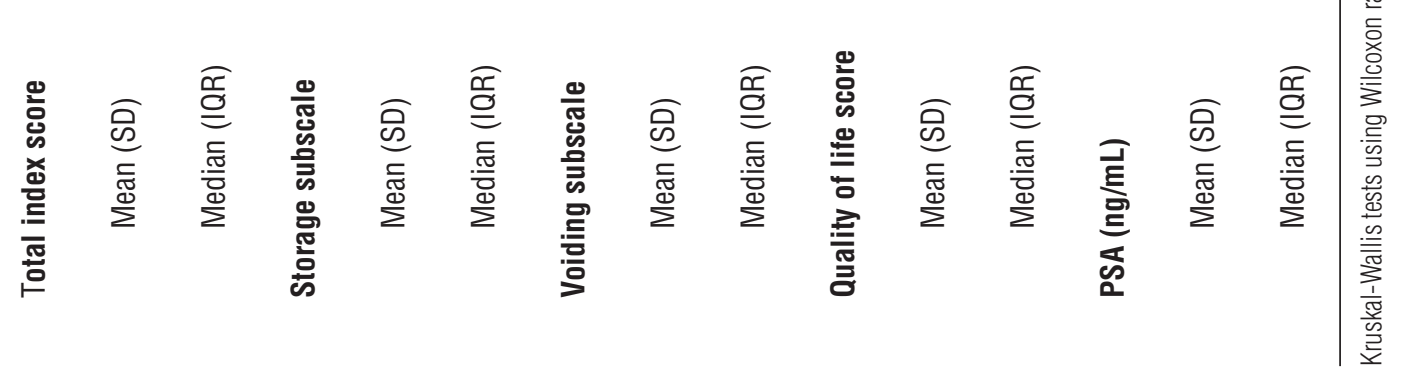


Table 4 - The association between inflammation grade and symptomatic improvement.

\begin{tabular}{|c|c|c|c|}
\hline & Improvement & No improvement & $p$-value \\
\hline \multicolumn{4}{|c|}{ Inflammation grade - most frequent } \\
\hline $0[n,(\%)]$ & $20(80)$ & $5(20)$ & 0.1363 \\
\hline I $[\mathrm{n},(\%)]$ & $44(73.3)$ & $16(26.7)$ & \\
\hline II $[\mathrm{n},(\%)]$ & $24(92.3)$ & $2(7.7)$ & \\
\hline \multicolumn{4}{|c|}{ Inflammation grade - most severe } \\
\hline $0[\mathrm{n},(\%)]$ & $9(81.8)$ & $2(18.2)$ & 0.3185 \\
\hline I $[\mathrm{n},(\%)]$ & $32(71.7)$ & $13(28.9)$ & \\
\hline II $[\mathrm{n},(\%)]$ & $41(83.7)$ & $8(16.3)$ & \\
\hline III [n, (\%)] & $6(100.0)$ & $0(0)$ & \\
\hline
\end{tabular}

Table 5 - Predictive factors of symptomatic improvement after alpha blocker therapy on univariate analysis.

\begin{tabular}{|c|c|c|c|}
\hline & \multicolumn{3}{|c|}{ Univariate analysis } \\
\hline & $\mathrm{OR}$ & $95 \% \mathrm{Cl}$ & $p$-value \\
\hline Age (years) & 0.97 & $0.91-1.04$ & 0.432 \\
\hline BMI & 1.12 & $0.95-1.31$ & 0.173 \\
\hline Treatment duration (months) & 0.92 & $0.86-0.99$ & $0.023^{*}$ \\
\hline PSA (ng/mL) & 1.08 & $0.94-1.24$ & 0.268 \\
\hline Inflammation grade - most frequent & 1.47 & $0.74-2.93$ & 0.271 \\
\hline Inflammation grade - most severe & 1.54 & $0.83-2.88$ & 0.174 \\
\hline \multicolumn{4}{|l|}{ TRUS } \\
\hline Total prostate volume (cc) & 1.00 & $0.97-1.03$ & 0.822 \\
\hline Transitional zone volume (cc) & 1.03 & $0.99-1.09$ & 0.167 \\
\hline \multicolumn{4}{|l|}{ IPSS } \\
\hline Total index score & 1.09 & $1.00-1.20$ & $0.047^{\star}$ \\
\hline Storage subscale & 1.06 & $0.90-1.25$ & 0.510 \\
\hline Voiding subscale & 1.14 & $1.02-1.29$ & $0.023^{*}$ \\
\hline Quality of life item & 1.25 & $0.83-1.89$ & 0.294 \\
\hline \multicolumn{4}{|l|}{ Uroflowmetry variables } \\
\hline Voided volume (mL) & 1.00 & $1.00-1.01$ & 0.202 \\
\hline Maximum flow rate $(\mathrm{mL} / \mathrm{sec})$ & 0.96 & $0.84-1.08$ & 0.484 \\
\hline Average flow rate $(\mathrm{mL} / \mathrm{sec})$ & 0.90 & $0.77-1.06$ & 0.205 \\
\hline Post-void residual urine $(\mathrm{mL})$ & 1.00 & $0.99-1.00$ & 0.224 \\
\hline
\end{tabular}


Table 6 - Predictive factors of symptomatic improvement after alpha blocker therapy on multivariate analysis.

\begin{tabular}{lccc}
\hline & \multicolumn{3}{c}{ Multivariate analysis } \\
\cline { 2 - 4 } & OR & $95 \% \mathrm{Cl}$ & $\mathrm{p}$-value \\
\hline Age (years) & 0.97 & $0.91-1.05$ & 0.462 \\
BMI & 1.15 & $0.95-1.40$ & 0.157 \\
Treatment duration (months) & 0.92 & $0.85-0.99$ & $0.025^{\star}$ \\
PSA (ng/mL) & 1.06 & $0.90-1.26$ & 0.477 \\
Inflammation grade - most frequent & & & \\
\multicolumn{1}{c}{ Grade I vs grade 0 } & 0.44 & $0.12-1.65$ & 0.223 \\
\multicolumn{1}{c}{ Grade II vs grade 0 } & 2.52 & $0.32-19.96$ & 0.382 \\
Total prostate volume (cc) & 0.99 & $0.95-1.02$ & 0.460 \\
IPSS & & & 0.826 \\
$\quad$ Storage subscale & & $0.79-1.21$ & $0.024^{*}$ \\
$\quad$ Voiding subscale & 0.98 & $1.02-1.34$ & \\
\hline
\end{tabular}

${ }^{\star} p<0.05$

REDUCE population, there was evidence of a weak relationship between the degree of LUTS and the degree of chronic inflammation (8). Collins et al. previously reported that prostatitis may be a risk factor for the development of pathologic prostatic hyperplasia into clinical prostatic hyperplasia (11). Additionally, inflammation detected on prostate biopsies performed during a baseline assessment in a subgroup of over 1000 patients enrolled in the MTOPS study predicted progression including symptom worsening, acute urinary retention, and the need for operative management in placebo-treated patients (12).

Basic and clinical research have sought to better elucidate the prostatic inflammation pathways and their relationship with BPH and prostate cancer, with a goal of identifying new therapeutic targets and strategies for reducing the risk of benign and malignant tumors of the prostate (13). Nickel raised the possibility of using anti-inflammatory agents as an additional treatment option for patients with BPH (6). Several reports have suggested that combination therapy of an alpha-blocker and an anti-inflammatory agent was more effective for treatment of BPH than monotherapy with an alpha blocker (14-16). Therefore, the management of intraprostatic inflammation plays an important role in the improvement of IPSS in patients with prostatic hyperplasia (17).

In this study, we found that lower grade inflammation was associated with higher MFR and AFR, and higher grade inflammation was related to larger prostate volume including total and transitional zone volume. As a result, higher grade inflammation may lead to increased prostate volume and subsequent increased severity of LUTS. However, caution must be taken in interpreting these results, as prostate volume itself had an association with LUTS. While greater prostate volume was associated with more severe LUTS, prostatic inflammation may also be an effect of the symptoms themselves, though it is difficult to distinguish direct and indirect causes of dysfunctional voiding. Furthermore, baseline IPSS and QoL scores were unrelated to prostatic inflammation grade. Similarly, Nickel et al. previously reported that higher average chronic inflammation scores were associated with higher IPSS, though there were no differences in IPSS or QoL scores prior to treatment among the three groups (8). Thus, subjective symptoms at baseline may not be associated with the degree of prostatic inflammation. 
Regarding factors that were predictive of alpha blocker treatment outcomes, more severe symptoms and shorter duration of treatment predicted improvement of symptoms. This is likely because symptom severity may not be directly related to the duration of treatment. We were unable to demonstrate a relationship between improvement of symptoms after alpha blocker treatment and inflammation grade.

This study was inherently limited by its retrospective design, though data were collected from 10 different hospitals to decrease the risk of bias. We included patients with PSA levels of $3-20 \mathrm{ng} / \mathrm{mL}$ and excluded one patient who was found to have prostate cancer on tissue biopsy. These inclusion criteria reflect those of a previous study by Kryvenko et al. That group analyzed the association between prostatic inflammation and pre-neoplastic lesions as risk factors for prostate cancer (18). They concluded that clinicians should consider patterns and extent of inflammation when managing high-risk patients with negative biopsy results. Therefore, the evaluation of pattern and extent of inflammation in prostate tissue has emerged as an important factor influencing treatment. Further studies will be required to confirm and extend these collective results.

\section{CONCLUSIONS}

Patients with high grade inflammation had lower urine flow rates and higher prostate volumes than patients with low grade inflammation. Prostatic inflammation grade did not affect outcomes of alpha blocker treatment. More severe symptoms, which were represented by high IPSS voiding scores, could be predictive of improvement of LUTS after treatment with an alpha blocker. Further studies will be required to investigate the causally related link between these findings.

\section{CONFLICT OF INTEREST}

None declared.

\section{REFERENCES}

1. Schatteman PH, Hoekx L, Wyndaele JJ, Jeuris W, Van Marck E: Inflammation in prostate biopsies of men without prostatic malignancy or clinical prostatitis: correlation with total serum PSA and PSA density. Eur Urol. 2000; 37: 40412.

2. Kramer G, Mitteregger D, Marberger M: Is benign prostatic hyperplasia $(\mathrm{BPH})$ an immune inflammatory disease? Eur Urol. 2007; 51: 1202-16.

3. Mishra VC, Allen DJ, Nicolaou C, Sharif H, Hudd C, Karim OM, et al.: Does intraprostatic inflammation have a role in the pathogenesis and progression of benign prostatic hyperplasia? BJU Int. 2007; 100: 327-31.

4. Kramer G, Marberger M: Could inflammation be a key component in the progression of benign prostatic hyperplasia? Curr Opin Urol. 2006; 16: 25-9.

5. Steiner GE, Newman ME, PaikI D, Stix U, Memaran-Dagda $\mathrm{N}$, Lee $\mathrm{C}$, et al.: Expression and function of pro-inflammatory interleukin IL-17 and IL-17 receptor in normal, benign hyperplastic, and malignant prostate. Prostate. 2003; 56: 171-82.

6. Nickel JC: Inflammation and benign prostatic hyperplasia. Urol Clin North Am. 2008; 35: 109-15.

7. Nickel JC, Downey J, Young I, Boag S: Asymptomatic inflammation and/or infection in benign prostatic hyperplasia. BJU Int. 1999; 84: 976-81.

8. Nickel JC, Roehrborn CG, O'Leary MP, Bostwick DG, Somerville MC, Rittmaster RS: The relationship between prostate inflammation and lower urinary tract symptoms: examination of baseline data from the REDUCE trial. Eur Urol. 2008; 54: 1379-84.

9. Irani J, Levillain P, Goujon JM, Bon D, Doré B, Aubert J: Inflammation in benign prostatic hyperplasia: correlation with prostate specific antigen value. J Urol. 1997; 157: 1301-3.

10. Boehm BJ, Colopy SA, Jerde TJ, Loftus CJ, Bushman W: Acute bacterial inflammation of the mouse prostate. Prostate. 2012; 72: 307-17.

11. Collins MM, Meigs JB, Barry MJ, Walker Corkery E, Giovannucci E, Kawachi I: Prevalence and correlates of prostatitis in the health professionals follow-up study cohort. J Urol. 2002; 167: 1363-6.

12. Roehrborn CG: Definition of at-risk patients: baseline variables. BJU Int. 2006; 97(Suppl 2): 7-11; discussion 21-2.

13. De Nunzio C, Kramer G, Marberger M, Montironi R, Nelson W, Schröder F, et al.: The controversial relationship between benign prostatic hyperplasia and prostate cancer: the role of inflammation. Eur Urol. 2011; 60: 106-17. 
14. Ozdemir I, Bozkurt O, Demir O, Aslan G, Esen AA: Combination therapy with doxazosin and tenoxicam for the management of lower urinary tract symptoms. Urology. 2009; 74: 431-5.

15. Di Silverio F, Bosman C, Salvatori M, Albanesi L, Proietti Pannunzi L, Ciccariello M, et al.: Combination therapy with rofecoxib and finasteride in the treatment of men with lower urinary tract symptoms (LUTS) and benign prostatic hyperplasia (BPH). Eur Urol. 2005; 47: 72-8; discussion 78-9.

16. Minnery $\mathrm{CH}$, Getzenberg $\mathrm{RH}$ : Benign prostatic hyperplasia cell line viability and modulation of jm-27 by doxazosin and Ibuprofen. J Urol. 2005; 174: 375-9.

17. Kwon YK, Choe MS, Seo KW, Park CH, Chang HS, Kim BH, et al.: The effect of intraprostatic chronic inflammation on benign prostatic hyperplasia treatment. Korean J Urol. 2010; 51: 266-70.
18. Kryvenko ON, Jankowski M, Chitale DA, Tang D, Rundle A Trudeau S, et al.: Inflammation and preneoplastic lesions in benign prostate as risk factors for prostate cancer. Mod Pathol. 2012; 25: 1023-32. Erratum in: Mod Pathol. 2013; 26: 464.

Correspondence address: Bong-Suk Shim, MD, PhD Department of Urology Ewha Womans University School of Medicine 911-1, Mok-Dong, Yangcheon-gu Seoul, 158-710, Korea Fax: +82 22 650-5157 E-mail: bonstone@ewha.ac.kr 